\title{
tic\&société
}

Vol. $1, n^{\circ} 1 \mid 2007$

De TIS à tic\&société : dix ans après

\section{Les téléphones portables, outils du dédoublement et de la densification du temps : un diagnostic confirmé}

Francis Jaureguiberry

\section{(2) OpenEdition \\ Journals}

Édition électronique

URL : http://journals.openedition.org/ticetsociete/281

DOI : 10.4000/ticetsociete.281

Éditeur

Association ARTIC

\section{Référence électronique}

Francis Jaureguiberry, «Les téléphones portables, outils du dédoublement et de la densification du temps : un diagnostic confirmé », tic\&société [En ligne], Vol. 1, n¹ | 2007, mis en ligne le 15 février 2008, consulté le 19 avril 2019. URL : http://journals.openedition.org/ticetsociete/281 ; DOI : 10.4000/ ticetsociete.281 


\section{Les téléphones portables, outils du dédoublement et de la densification du temps : un diagnostic confirmé}

Francis Jauréguiberry, Professeur des Universités, Directeur du laboratoire Société Environnement Territoire (UMR 5603 du CNRS)

Adresse postale :

IRSAM

avenue du Doyen Poplawski

64000 Pau

France

Adresse de courriel :

francis.jaureguiberry@univ-pau.fr

Francis Jauréguiberry est sociologue, professeur à l'Université de Pau et directeur du laboratoire SET (Société Environnement Territoire) du CNRS. Ses recherches portent sur les nouvelles formes d'identité et de sociabilité générées par l'extension des technologies de l'information et de la communication. Derniers ouvrages: Les branchés du portable. Sociologie des usages, Paris, PUF, 2003 et, avec Serge Proulx, L'Internet, nouvel espace citoyen?, Paris, l'Harmattan, 2003. 
Les téléphones portables, outils du dédoublement et de la densification du temps:

un diagnostic confirmé

\begin{abstract}
Résumé
L'originalité de la rentabilisation du temps permise par l'usage des téléphones portables réside dans le fait qu'elle est obtenue non seulement de façon classique, par la densification du temps grâce à une meilleure organisation des taches dans leur déroulement et leur succession, mais aussi de façon inédite, par le dédoublement du temps grâce à la superposition simultanée d'un temps médiatique à un temps physique. Ce phénomène, déjà repéré il y a dix ans, n'a cessé depuis de se développer en entraînant un raccourcissement des délais, une accélération du temps et une multiplication des situations d'urgence. Mais, face à la dispersion et à l'égarement que ce phénomène peut engendrer, à l'aléatoire trop souvent côtoyé et à l'éphémère renouvelé dans une sorte d'éternel présent, une réaction n'a, parallèlement, cessé de se développer. Elle renvoie à une logique d'action critique qui vise à ne pas se laisser déposséder de sa propre temporalité, de ses propres rythmes au profit d'une mise en synchronie généralisée, à réintroduire l'épaisseur du temps de la maturation, de la réflexion et de la méditation là où le heurt de l'immédiat et de l'urgence oblige trop souvent à réagir sous le mode de l'impulsion.
\end{abstract}

Mots-clé : Téléphone portable, sociologie des usages, gestion du temps, urgence, immédiat.

\begin{abstract}
The originality of time optimization afforded by the use of mobile telephones is due to the fact that,not only is time condensed through better organization in the sequencing and processing of tasks, but also through the doubling of time with the simultaneous superposition of media time and physical time. Since its identification ten years ago, this phenomenon has continued to develop and has brought with it shortened delays, acceleration of time and the multiplication of emergency situations. However, we also witness the parallel development of reactions to this dispersion and getting lost, to too much randomness and to continually renewed ephemerality in the present. It finds its source in a logic of critical action which refuses dispossession of one's own temporality and rhythms for generalized synchonization, and which reintroduces the thickness of mature time, reflection and meditation to counteract pressures for immediate, urgent reactions by impulse.
\end{abstract}


Francis JAUREGUIBERRY

Key words : mobile telephone, sociology of uses, time management, urgency, immediacy

\section{Resumen}

El uso de los teléfonos móviles permite una rentabilización temporal de manera clásica, a través de la densificación del tiempo mediante una mejor organización de las tareas y de su secuenciación. No obstante, la originalidad de esta rentabilización temporal reside en el desdoblamiento del tiempo, gracias a la superposición simultánea de un tiempo mediático sobre un tiempo físico. Este fenómeno, ya investigado hace diez años, no ha cesado de amplificarse e implica una compresión de los plazos y una aceleración del tiempo, así como una multiplicación de las situaciones de emergencia. Ahora bien, frente a la dispersión y a la incertidumbre que este fenómeno pueden generar, y frente a la aleatoriedad resultante y a lo efímero - que continuamente se renueva en una especie de eterno presenteno ha dejado de desarrollarse una cierta reacción. Esta reacción es una lógica de acción crítica que no permite ser despojada de su propia temporalidad y de sus propios ritmos, en aras de una sincronía generalizada. Lleva también a reintroducir el espesor del tiempo de la maduración, de la reflexión y de la mediación, precisamente allí en donde la premura de lo inmediato y de la urgencia obligan, a menudo, a responder de modo impulsivo.

Palabras-clave : telefono móvil, sociología de los usos, gestión del tiempo, urgencia, inmediatez. 
Les téléphones portables, outils du dédoublement et de la densification du temps : un diagnostic confirmé

La revue tic\&société qui prend le relais de TIS, dix ans après - et il faut en féliciter ses nouveaux promoteurs - me demande de revenir sur un article paru dans cette même revue il y a plus de dix ans de cela ${ }^{1}$. Revisiter un de ses textes est toujours délicat. Quiconque écrit connaît le plaisir d'abandonner un texte et aussi... la difficulté à revenir dessus. Aussi, et comme nous y invite Montaigne ${ }^{2}$, je ne corrigerai rien de ce texte mais me contenterai d'ajouter quelques remarques nées de plusieurs enquêtes et observations ultérieures ${ }^{3}$.

\section{1- Le dédoublement du temps}

Un des premiers constats de l'article de 1996 montrait que les portables étaient un outil de réaménagement du temps vers sa plus grande rentabilisation. S'appuyant sur de nombreux exemples, l'article montrait comment l'originalité de ce réaménagement résidait dans le fait qu'il était obtenu non seulement de façon "classique ", par la densification du temps grâce à une meilleure organisation des tâches dans leur déroulement et leur succession, mais aussi de façon inédite, par le dédoublement du temps grâce à la superposition simultanée d'un temps médiatique à un temps physique.

Le temps physique «doublé » est en général vacant, interstitiel ou "mal utilisé » selon une logique rentabiliste. II s'agit par exemple du temps contraint des trajets physiques durant lesquels « on est bloqué à ne rien faire ", de celui des attentes dues à une affluence, à un retard ou à un contrordre, mais aussi de celui qui s'avère non conforme, en utilité ou en intensité, à ce que l'on avait projeté. Le téléphone portatif permet de s'extraire de ces temps contraints,

\footnotetext{
1 «De l'usage des téléphones portatifs comme expérience du dédoublement et de l'accélération du temps ", Technologies de l'information et Société (TIS), vol. 8, n², 1996, pp. 169-187. Consultable en ligne : <http://revuetis.mshparisnord.org/article.php3?id_article=144>.

${ }_{2}$ « J'ajoute, mais je ne corrige pas. Premièrement parce que celui qui a hypothéqué au monde son ouvrage, je trouve apparence qu'il n'y ait plus droit. Qu'il dise, s'il peut, mieux ailleurs, et ne corrompe la besogne qu'il a vendue... Secondement parce que, pour mon regard, je crains de perdre au change : mon entendement ne va pas toujours avant, il va à reculons aussi. Je ne me défie guère moins de mes fantaisies pour être secondes ou tierces que premières, ou présentes que passées. Nous nous corrigeons aussi sottement souvent que nous corrigeons les autres", MONTAIGNE, Essais, PUF, 1965, pp. 963-964.

${ }^{3}$ Les citations du texte de 1996 apparaissent en alinéa.
} 


\begin{abstract}
«presque morts». Ou, plus exactement, il offre la possibilité de leur superposer un second temps médiatique, plus utile et donc rentable, sans pour autant « assassiner» totalement (pour rester dans la métaphore) les premiers : on continue de participer (à son volant) à la circulation automobile et on échange simultanément (à son téléphone portable) des informations avec son secrétariat. II ne s'agit donc plus simplement de remplacer une occupation par une autre ou d'accélérer leur succession, mais de les superposer simultanément (Jauréguiberry, 1996, p. 171).
\end{abstract}

Ce phénomène de dédoublement du temps m'apparaissait central dans l'expérience des premiers utilisateurs du portable. Quelques années plus tard, alors que l'usage des portables s'étendait à l'ensemble de la population, je me suis posé la question de savoir si cette dimension de dédoublement du temps en vue de sa rentabilisation n'était pas un travers dû à l'appartenance socioprofessionnelle des premiers utilisateurs qui étaient essentiellement des dirigeants, cadres, membres de professions libérales ou professionnels de l'urgence. Responsables d'entreprises ou de structures engagées dans une chrono compétitivité générale (où, toutes choses étant égales par ailleurs, c'est celui qui réagit le premier qui l'emporte), pris dans des emplois du temps tendus, soucieux de ne pas perdre leur temps, ces premiers utilisateurs ont d'entrée converti les portables en instruments de rentabilisation de leur temps. Outils du «temps réel » (immédiateté), les portables sont employés à lutter contre la réalité du temps qui résiste sous forme de lenteur, de retards, de temps morts ou de délais. Mais cette dimension, centrale dans les usages observés entre 1994 et 1996 alors que le nombre d'utilisateurs était inférieur à 2 millions (en France), est-elle toujours valable ou tout au moins aussi importante à partir du moment où le nombre d'usagers explose (passant de 3,5 millions en juin 1997 à 37,8 millions cinq ans plus tard, le taux d'équipement grimpant dans le même temps de $3,7 \%$ à $62,7 \%)^{4}$ ?

Procédant à trois autres terrains en 2000, 2003 et 2005 et prenant soin de constituer mes panels et groupes d'intervention sociologique de façon à ce qu'ils soient représentatifs de l'ensemble des usagers, je fus surpris de voir à quel

\footnotetext{
${ }^{4}$ En septembre 2007 en France, on compte 52 millions d'utilisateurs et le taux d'équipement est de $83 \%$.
} 
Les téléphones portables, outils du dédoublement et de la densification du temps : un diagnostic confirmé

point cette dimension de dédoublement du temps en vue de sa rentabilisation était transversale aux catégories socioprofessionnelles, aux sexes et (avec la nuance apportée plus loin) aux âges. Bien sûr les contenus des occupations superposées diffèrent grandement, mais le processus de dédoublement du temps en vue de sa densification est bien le même. Par exemple, la façon dont les femmes se servent du portable (elles étaient encore ultra minoritaires parmi les utilisateurs en janvier 1996 : 5 \% seulement, mais à quasi-parité lors de mes derniers terrains) rappelle curieusement celle des cadres supérieurs ! Souvent contraintes d'organiser un ensemble d'occupations de nature très hétérogène (rendez-vous professionnels, garde des enfants, gestion du foyer, maintien du cercle d'amis, etc.), les femmes utilisent le portable dans un même but de coordination, de fluidification et de rentabilisation du temps. On observe la même logique chez les employés de bureaux, les artisans, les enseignants, etc.

Un autre aspect de ce dédoublement, absent lors de la rédaction de l'article de 1996, est ensuite apparu avec force : celui de la fuite du temps perdu. Ici, le portable ne sert pas à organiser l'existant dans son excès, mais à le fuir dans son vide. Pour le dire simplement : il est des moments où l'individu s'ennuie. Dans une file d'attente, lors d'un trajet ou seul à la table d'un restaurant, il fait l'expérience du vide creusé par la réalité du temps en regard des attentes, qu'idéalement et narcissiquement, il se fixe en accord avec l'injonction contemporaine à vivre de façon continue une existence dense et trépidante. Le portable permet à l'individu de se déprendre de ce sentiment de déception ou tout au moins d'ennui en le plongeant dans le temps superposé des télécommunications. On observe alors des attitudes compulsives de manipulation du portable : multiplication les appels, consultation de façon fébrile des boîtes vocales, vérification de la bonne couverture de la zone, et, depuis peu, consultation ou envoi d'emails et surf sur Internet. Cette dimension est particulièrement présente chez les plus jeunes.

De façon plus fondamentale, l'immédiateté télécommunicationnelle rendue possible par les portables nous révèle une des dimensions désormais centrales du modèle culturel de nos sociétés hyper modernes : la gestion rentabiliste de sa vie. De façon diffuse, les modes d'action repérables dans la sphère économique (pragmatisme, utilitarisme, compétition, rentabilité, efficacité, désir de gains et de puissance) sont appliqués à ce qui est devenu la gestion des occupations et relations privées. La vie est un capital qu'il s'agit de faire fructifier au plus vite. Des profits à court terme sont attendus! Le scénario inacceptable n'est pas, comme dans la sphère professionnelle, la défaite économique, mais 
Francis JAUREGUIBERRY

l'occasion ratée. Mû par le désir de réussir sa vie dans tous ses instants, porté par un souci de performance et d'intensité, l'individu contemporain est constamment à la recherche du «mieux ». À un moment où la rédemption religieuse et les utopies sociales ne parviennent plus à définir un au-delà d'espérance producteur d'identifications et de mobilisations, l'individu n'a plus d'autre horizon que sa propre vie qu'il lui faut dès lors réussir à tout prix (Ehrenberg, 1995). Démiurge de lui-même, gestionnaire de ses propres ressources, la raison de son existence se mesure alors à l'aune de l'intensité que cette même existence lui procure. La subjectivité individuelle semble alors l'emporter sur toute détermination sociale : l'idéal de soi que se fixe l'individu est toujours un dépassement de soi dans l'intensité. Le portable accompagne parfaitement cette recherche du potentiellement mieux contre le certainement moyen. II s'agit d'être à la fois en situation de ne rien rater, c'est-à-dire à l'écoute (branché) et en disposition de commuter immédiatement (zapper) sur ce qui apparaît subitement mieux ou plus intense.

\section{2- Ecrasement du temps sur l'immédiat et zapping occupationnel}

Dans l'article de 1996, j'observais (paragraphe 2 : «La relativisation du déjà prévu » et paragraphe $4:$ "La distorsion du temps ») ${ }^{5}$ certaines conséquences d'une telle «gestion à flux tendus » de sa propre vie.

L'appétence du mieux, doublée de cette nouvelle possibilité de prévenir immédiatement ses interlocuteurs d'un changement de programme, conduit à une logique de l'alternative permanente. Une singulière alchimie apparaît alors, mêlant, en les opposant souvent, désirs et devoirs, raison et sentiments, pouvoir et morale, la pierre philosophale étant en la matière le bon choix absolu : celui qui n'aura aucun arrière-goût de nostalgie... Cette logique de l'alternative, doublée des tensions qu'elle suscite, contribue au renforcement d'un environnement où tout devient potentiellement précaire. Les agendas s'ouvrent quotidiennement sur des futurs dont beaucoup se trouvent transformés, avant même

\footnotetext{
${ }^{5}$ Dix ans après, le thème du paragraphe 3 (le stress de la déconnexion dû à une mauvaise couverture hertzienne de la zone, à une panne ou à un oubli du téléphone portatif) m'apparaît désuet.
} 
Les téléphones portables, outils du dédoublement et de la densification du temps : un diagnostic confirmé

d'avoir existé, en passés antérieurs par un coup de fil de dernière minute (p. 172).

Je n'ai pas cessé, depuis, d'observer le renforcement de cette propension à la relativité et à l'alternative permanente. À la merci de zaps impulsifs ou de dernière minute, les emplois du temps deviennent à la fois plus élastiques, plus imprévisibles et plus heurtés. Dans l'attente de l' «advenance » et dans l'espérance du meilleur, le quotidiennement certain et l'acquis moyen paraissent potentiellement fades, sont questionnés et relativisés (Bauman, 2004). Le flou qui en résulte peut certes dérouter, mais il est aussi gage que «tout est possible ». Et lorsqu'un «possible» advient, les changements sont d'autant plus précipités qu'ils peuvent être médiatisés immédiatement, quel que soit le lieu, par un appel téléphonique. Ce qu'il importe de souligner dans ce cas n'est pas tant le contenu de ce changement - qui, dans la plupart des cas, porte plutôt à sourire - que l'extension d'une sorte de propension à la versatilité qu'elle accompagne.

Tant que les situations agréables et les résultats positifs s'accumulent, ce type de conduite est positivement vécu par l'acteur. II correspond même assez bien à l'image idéale que certains se font de la société postmoderne « de l'information et de la communication ». Libre sur un marché libre, l'individuzappeur se composerait une vie à la carte dont la programmation n'aurait d'autre objet que la satisfaction la plus immédiate de ses désirs et attentes. Les opportunités et choix se multipliant, la vie deviendrait une sorte de surfing jouissif et parfois esthétique sur des situations qui, à l'image des vagues, seraient à la fois éphémères et constamment renouvelées dans une sorte de mouvement perpétuel. En regard des idéologies et utopies collectives du siècle passé, que trop de mouvements ont cherché à imposer avec les errements que l'on sait, le caractère éphémère et individuel des passions post-modernes serait le meilleur garant des libertés ${ }^{6}$.

Toutefois, si le zapping peut conduire à cette sorte d'intensité jouissive et à une dispersion légèrement enivrante, il peut aussi comporter des dangers. En note du paragraphe cité, j'indiquais sans développer que :

Si l'on accepte que le déroulement du temps et la croyance en l'avenir sont bien des marques de la modernité (en regard du temps cyclique et des modes

\footnotetext{
${ }^{6}$ Pour un développement optimiste de cette vision, voir par exemple Lipovetsky $(1983,1987)$.
} 


\begin{abstract}
de reproduction des communautés traditionnelles), et que les principaux outils de la rationalisation de ce temps ont été la prévision, la planification et l'exactitude, on est en droit de se poser la question de la rationalité du développement de certains modes de réaction à l'urgence et à l'aléatoire. II n'est en effet pas certain que le passage d'une logique de la planification faisant usage de l'exactitude à une logique de l'opportunité productrice de précarité (par le biais des décisions de dernière minute et à court terme) soit synonyme d'extension de la rationalisation de l'existence. Trop de planification et d'exactitude figent sans doute le social au point de freiner la créativité des individus, mais trop d'opportunisme et d'aléas risquent de rendre l'action individuelle imprévisible et la société incertaine (p. 185, note 3 ).
\end{abstract}

Avec du recul, cette intuition me paraît valable car c'est la notion même de projet qui semble questionnée. Un projet nécessite une certaine confiance dans l'avenir. II relève d'un pari sur le futur en pensant que l'action programmée pourra s'y déployer. II n'y a évidemment, en la matière, aucune certitude : seule la confiance permet de différer, de planifier, de se représenter ce qui sera contre ce qui est. Mais si le présent lui-même apparaît comme indéterminé, n'est plus vécu que comme immédiateté éphémère, comment penser le projet dans sa durée ? Dans un monde où il faut s'adapter rapidement, comment peut-on encore adapter le monde dans la durée ${ }^{7}$ ?

\title{
3- L'urgence, rançon de l'immédiat
}

Sous le titre de «la contagion de l'urgence » (paragraphe 5), je décrivais en 1996 la montée de l'urgence comme une « maladie » dont les portables étaient un des principaux vecteurs de contagion. Quatre ans après, dans un autre article (Jauréguiberry, 2000), je précisais cette idée et notais en particulier que :

\footnotetext{
7 Zaki Laïdi (2000) souligne les dangers qu'une telle absorption du futur par le présent représente pour la démocratie. Le politique, pensant renouveler sa légitimation en se mettant à l'écoute de l'immédiat, perd son pouvoir visionnaire producteur de projet pour le futur au profit d'un rôle gestionnaire destiné à surmonter des contraintes du présent. La réduction de la distance entre gestion de l'immédiat et représentation du futur accompagne la chute des catégories de l'espoir, de l'attente ou de l'utopie politique.
} 
Les téléphones portables, outils du dédoublement et de la densification du temps : un diagnostic confirmé

C'est parce qu'il y a possibilité de réagir jusqu'au dernier moment que de plus en plus de gens s'inscrivent dans des scénarios limites. Ils calculent en effet toujours plus juste sachant que, si ça ne passe pas, il y aura toujours le recours ultime d'un « appel urgent » pour « réparer » leur retard, de la même façon que certains alpinistes, assurés qu'ils pourront immédiatement déclencher des secours en cas d'accident, s'engagent dans des voies manifestement trop dures pour eux. Ce type d'appel ne devient heureusement pas la règle, mais le raccourcissement des délais et la prise de risques, oui.

Le développement de cette notion de scénario limite m'a par la suite permis de travailler sur le concept même d'urgence et d'en forger une définition que j'ai depuis souvent reprise ${ }^{8}$. L'urgence naît toujours d'une double prise de conscience : d'une part, qu'un pan incontournable de la réalité relève d'un scénario aux conséquences dramatiques ou inacceptables et, d'autre part, que seule une action d'une exceptionnelle rapidité peut empêcher ce scénario d'aller à son terme. L'une ne va pas sans l'autre, ou alors il ne s'agit plus d'urgence. Ce n'est pas parce qu'une action est exceptionnellement rapide qu'elle est urgente. Par exemple, les courses (compétitions) sont par définition toujours rapides, mais rarement urgentes. De même, ce n'est pas parce qu'un scénario catastrophe est en marche qu'il génère ipso facto de l'urgence: le laps de temps laissé à la réaction peut être suffisamment long pour que celle-ci ne soit pas vécue sous la forme d'une rapidité imposée. Cette double prise de conscience déclenche un compte à rebours qu'il s'agit d'arrêter illico presto. Dit autrement : on court à la catastrophe si rien n'est immédiatement mis en œuvre. Le scénario à la base de l'urgence est toujours menaçant, néfaste ou fatal. S'il n'était pas appréhendé comme l'irréversibilité d'une négativité mais d'une positivité, il s'agirait non plus d'urgence mais d'attente ou d'impatience, le « vivement » remplaçant en la matière le « vite ».

L'urgence apparaît donc comme un piège du temps. La dangerosité de ce piège dépend de l'interprétation qui en est faite, puisque l'urgence naît toujours de la double prise de conscience qui vient d'être évoquée. Les notions de danger et de rapidité étant relatives, il s'ensuit que ce qui est urgent pour certains ne l'est pas pour d'autres. Tout dépend du système de référence des

\footnotetext{
${ }^{8}$ Elle a aussi été reprise par Nicole Auber dans son livre consacré au culte de l'urgence (2003).
} 
Francis JAUREGUIBERRY

acteurs, de leur subjectivité et de leurs capacités cognitives. Mais, quelles que soient les disparités que l'on peut observer entre individus, la ligne de l'urgence est toujours franchie lorsque la notion de piège du temps apparaît clairement. Or, force est de reconnaître que, globalement, l'immédiateté télécommunicationnelle est en train d'accroître la surface de cette notion. En quelques années seulement, le processus d'accélération et de densification du temps, à l'œuvre depuis deux siècles, s'est en effet vu doublé par une nouvelle donnée : celle que les informaticiens ont nommée «temps réel ", synonyme d'immédiateté et de simultanéité. À peine remises des bouleversements que la rationalisation et l'instrumentalisation du temps ont opérés en leur sein, nos sociétés sont confrontées à un nouvel impératif : il leur faut désormais réagir à l'instant. Un espace sans distance et un temps sans délais se superposent peu à peu à l'espace-temps classique.

En nous accompagnant partout, le portable permet de très facilement prétexter un aléa de dernière minute pour annuler un rendez-vous. Or, force est de constater que, pour beaucoup, avancer une "fausse urgence" est une façon " en voie de développement » afin de "jouer au mécano avec un emploi du temps ${ }^{9}$. Ceci signifie rien moins que l'urgence (en général) a acquis un statut social suffisamment prégnant pour qu'elle puisse être spontanément déclinée et surtout acceptée comme excuse. II devient «normal » d'être pris par une urgence et de devoir ainsi être obligé d'annuler un rendez-vous sans autre formalité. Bien entendu, le degré de liberté pris envers un correspondant est directement proportionnel à celui de son pouvoir envers lui. Mais cela ne relativise que modérément le poids du constat : l'urgence l'emporte souvent sans qu'elle ait désormais besoin d'autre justification qu'elle-même.

La chasse au temps perdu, le culte du potentiel, la montée de l'urgence, la pression de l'immédiat et la multiplication des informations plongent l'individu dans une situation d'injonctions répétées à réagir de plus en plus vite. Cette accélération peut être vécue de façon positive : comme multiplicateur d'activités et d'opportunités, comme révélateur de certaines potentialités organisationnelles jusqu'alors inexploitées, comme agent de simplification ou encore comme réhabilitation de l'intuition individuelle dans la gestion des affaires. Elle peut aussi être source de satisfactions, en particulier pour certains professionnels indépendants qui, vivant cette course au temps sous la forme de défis renouvelés, la trouvent passionnante et parfois gratifiante. Mais cette

\footnotetext{
${ }^{9}$ Nous ne traiterons pas ici de l'aspect moral de l'affaire... Notons simplement que la célébration du toujours plus et du toujours mieux semble se muer en règle de conduite dont l'opportunisme se nourrit aux dépens du devoir que plus aucun fondement n'alimente. Sur ce thème, voir Zygmunt Bauman (2003).
} 
Les téléphones portables, outils du dédoublement et de la densification du temps :

un diagnostic confirmé

accélération peut aussi donner le vertige, et la chute n'est alors pas exclue. Dans un monde où tout s'accélère et se bouscule, le branché, placé en état d'urgence quasi permanent court en effet deux risques.

Le premier de ces risques est de réagir à l'impulsion afin d'éviter ce qu'on pourrait appeler «l'effet bouchon » : l'accumulation incontrôlable d'informations interdisant leur traitement efficace. Face à une pile de notes, à une succession d'emails urgents et à un sans fin de sollicitations téléphoniques, il faut aller vite. Non seulement il faut toujours être connecté, mais il faut aussi pouvoir répondre rapidement. Dans cette accélération, synonyme de diminution du temps de réflexion, l'accessoire risque de recouvrir l'essentiel. Outre le stress lié à l'activité fébrile qui en résulte, le danger d'une telle réaction est de voir remplacer la réflexion et l'imaginaire par une espèce de réflexe à parer au plus pressé. Le branché se convertit en pompier cherchant à éteindre le feu de l'urgence là où il prend. Le coup de fil a dès lors priorité sur la personne présente, le courrier électronique sur le courrier postal, et le beeper arrête tout, séance tenante. Comme si l'individu ou l'organisation se mettaient aux ordres de l'urgence, l'advenant supplantant l'existant. Focalisées sur la réaction aux sollicitations immédiates, personnes ou structures courent alors le risque de perdre tout pouvoir stratégique au profit de pures tactiques d'adaptation à un environnement qu'elles ne maîtrisent plus. Dans ce cas, l'information devient bruit, la vitesse précipitation, et les passages à l'acte font office de décisions. D'actif et réfléchi, le choix devient réactif et improvisé, et a donc toute chance d'être dépendant.

Le second risque est de se mettre à hésiter dans l'urgence. Les prises de décision deviennent alors autant de violences que l'individu s'impose dans une situation qu'il ne maîtrise plus. Tensions, stress et parfois même anxiété ont alors toutes chances d'apparaître. Le branché se sent non plus interpellé mais harcelé. Tout un ensemble de pathologies psychosomatiques sont associées à cette contradiction dans laquelle se place l'individu en " surchauffe » occupationnelle. D'un côté la conscience de l'urgence, de l'autre celle de ne pouvoir y faire face, ce qui ne fait qu'augmenter la pression. II est même des cas où l'individu reste comme " sonné » devant trop d'interpellations. Pour lui, la seule façon de ne pas cesser d'exister complètement, est alors de 
Francis JAUREGUIBERRY

" craquer ». Entrant dans un véritable état de catalepsie, il « démissionne » par overdose communicationnelle pour tomber dans un vide apathique ${ }^{10}$.

\section{4- Le tourbillon du zappeur}

Cherchant à mieux cerner les effets pervers de cet écrasement du temps sur l'immédiat et de cette montée de l'urgence, je proposais, en paragraphe 6 , la notion de « syndrome du zappeur ».

J'appelle «syndrome du zappeur » l'ensemble des symptômes du mal latent qui guette ceux qui vivent de façon trop entière une expérience d'ubiquité médiatique au point de s'y faire absorber. C'est tout à la fois l'insatisfaction du mieux potentiel introuvable, l'anxiété du temps perdu et celle du dernier moment, le désir jamais assouvi d'être ici et ailleurs en même temps, la peur de rater quelque chose d'important et de ne pas pouvoir tout faire, le stress des choix hâtifs et l'insatisfaction qui en découle, la confusion due à une surinformation éphémère, la tension entre une instrumentalisation croissante du rapport aux autres et une recherche de sentiments profonds (p. 178).

Après la «maladie " de l'urgence, je continuais donc à filer la métaphore médicale. Avec du recul, je ne suis pas certain que cette façon de désigner comme pathologiques des pratiques soit une bonne chose. Mes recherches m'ont amené à remplacer peu à peu la notion de syndrome par celle de tourbillon, à la fois plus neutre, expressive et proche du témoignage des personnes interrogées (plusieurs ayant spontanément employé ce mot).

Le tourbillon du zappeur est synonyme d'emballement occupationnel et d'agitation désordonnée. Comme sous l'effet d'une force centrifuge due à une accélération non maîtrisée, l'individu semble dépossédé du sens de son action. Contraint à réagir sur le mode de l'urgence à une masse d'information grandissante et à des aléas de plus en plus fréquents, il se trouve réduit à s'accrocher où il peut s'il ne veut pas sombrer, à «faire des coups ", à développer des tactiques de nature opportuniste. L'acteur est alors moins mû

\footnotetext{
10 Sur ce thème, voir Nicole Aubert (2003). Toute «gestion en temps réel » ou tout «pilotage en situation d'urgence » ne saurait évidemment conduire à une telle situation. Non seulement parce que les réactions à l'urgence peuvent avoir été planifiées de façon préalable (on parle alors de «procédures», fruits d'anticipations stratégiques), mais aussi, de façon plus fondamentale, parce que ces réactions peuvent relever d'une rationalité, certes limitée par le temps, mais non hétérogène à une continuité stratégique pourvoyeuse de sens.
} 
Les téléphones portables, outils du dédoublement et de la densification du temps : un diagnostic confirmé

par une logique de type stratégique, visant à adopter les meilleurs moyens par rapport à une fin qu'il s'est fixée, que par une logique de type tactique, visant à s'adapter au mieux à une situation qu'il ne domine plus. Ce n'est pas parce qu'il fait beaucoup de choses (y compris à la fois) ou va très vite qu'un individu tombe dans le tourbillon du zappeur, c'est parce qu'il expérimente une trop grande différence entre ses capacités cognitives, d'action ou de réflexion et ce qu'exigent comme réponses les situations qu'il traverse. C'est lorsque cette différence paraît insurmontable qu'apparaissent stress et agitation. II s'agit donc d'une notion relative, propre à chaque individu.

La mise en évidence de ce tourbillon du zappeur, figure extrême de l'individu branché, a méthodologiquement pris une grande importante dans mes recherches ultérieures. Elle m'a permis de donner aux groupes des interventions sociologiques que j'ai depuis menées (mais aussi lors d'interviews individuelles) l'image de ce que peut devenir l'expérience de zapping médiatique lorsqu'on s'y absorbe trop. En faisant fonctionner le tourbillon du zappeur comme un idéal-type, j'ai pu définir avec les personnes que j'interviewais la plus ou moins grande distance qui les en séparait. Le mesure de cet écart et le récit de l'attirance ou au contraire de la répulsion que le tourbillon suscitait chez elles a grandement contribué à dégager une forme de connaissance. Avant la rédaction de l'article de 1996, je n'avais rencontré aucune personne étant tombé dans ce tourbillon. Et je pouvais écrire qu'

aucun des participants ne s'y est totalement reconnu.

Plusieurs ont même déclaré en être très éloigné.

Pourtant, tous ont admis le connaître d'une certaine façon et surtout, tous le tiennent pour un danger potentiel. Le syndrome du zappeur est ce qu'il faut éviter : voilà le constat unanime des participants à nos groupes. «On a tous un pied dedans, il faut éviter d'y mettre les deux » (p. 179).

Depuis, j'ai rencontré plusieurs «noyés », mais n'en ai pas fait un sujet d'étude. J'ai par contre continué à faire fonctionner cet idéal-type comme un repère d'analyse. Grâce à lui, il devient beaucoup plus facile de lire les conduites liées à l'expérience de zapping médiatique et occupationnel, et de leur donner un sens. Dit de façon lapidaire : toutes visent une pleine expérience de dédoublement et de densification du temps sans pour cela courir le risque de tomber dans le tourbillon du zappeur. Mais ces conduites sont hétérogènes. Elles peuvent être classées en trois : le refus, la fuite en avant, et la ruse. Déjà repérées en 1996, je les exposerai plus loin. 
Francis JAUREGUIBERRY

\section{5- Retrouver le temps}

Le tourbillon du branché-zappeur attire. II attire parce que la force du courant qui l'alimente est celle du système économique actuel basé sur la généralisation de la gestion en «temps réel » et qu'il est difficile d'échapper à sa pression. II attire aussi parce que la forme qu'il donne à l'action (la vitesse, le défi, le zapping, le sentiment de puissance) n'est pas sans procurer une certaine jouissance à ceux qui l'expérimentent. II attire enfin peut-être parce qu'en ramassant le temps dans une sorte de présent continu, il "distrait » (au sens pascalien du terme) l'individu du temps qui passe, et donc de questions existentielles potentiellement inquiétantes ${ }^{11}$ (p. 181).

Mais contre cette attirance, j'indiquais qu'une réaction apparaissait et pouvait être observée :

Face à la dispersion et à l'égarement qu'il peut engendrer, à l'aléatoire trop souvent côtoyé et au stress qu'il suscite, à l'éphémère renouvelé dans une sorte d'éternel présent, une réaction apparaît. Elle renvoie à une logique critique qui vise à ne pas se laisser déposséder de sa propre temporalité, de ses propres rythmes au profit d'une mise en synchronie universelle qui unirait «en temps réel " tous les "branchés » du "grand réseau » dans une sorte de compulsion totalisante. Cette logique critique déroule ce que l'urgence ficelle. Elle résiste à l'idée que la vie ne serait qu'une suite d'instants et d'événements sans liens entre eux. Elle rétablit la durée et replace l'individu dans une continuité qui lui permet de renouer avec un ordre possible de référence. Elle réintroduit l'épaisseur du temps de la maturation, de la réflexion

\footnotetext{
11 Si l'on accepte en effet, avec Heidegger, que c'est parce qu'il "s'insère dans le temps " (Zeitigung) que l'individu a conscience de son " être-là » (Dasein), et que cette conscience est avant tout celle de sa finitude individuelle (l'être est dans la conscience du temps qui est celle de sa mort), on peut penser que l'écrasement du présent sur l'immédiat est aussi une façon d'échapper à l'angoisse qu'une telle conscience entraîne.
}

tic\&société - 1(1), 2007 
Les téléphones portables, outils du dédoublement et de la densification du temps : un diagnostic confirmé

et de la méditation là où le heurt de l'immédiat et de l'urgence oblige à réagir trop souvent sous le mode de l'impulsion (pp. 181-182).

Cette logique est celle de la mise en perspective de soi dans le temps sous la forme d'un récit, d'une narration sans cesse revisitée. Car, en définitive, ce n'est pas le temps qui passe mais l'individu. Le temps que l'on découvre alors est celui du passé, du souvenir et du retour sur soi. C'est aussi le temps de l'anticipation, de la crainte ou de l'espoir. La possibilité, grâce au portable, de pouvoir joindre immédiatement son interlocuteur pour lui faire part d'une intuition, d'une idée ou d'un élan sentimental est sans doute une chance. Mais elle peut aussi représenter un danger pour l'émotion pensée non plus comme impulsion mais comme tension créatrice. Le risque réside dans le fait de voir l'impulsion chasser l'imagination, et le bavardage remplacer l'échange. Le silence et le différé, condition de retour sur le passé et de projection sur l'avenir, sont les complices d'un présent créateur. Mais, lorsque ce présent n'est plus qu'une succession d'immédiats éphémères, où se situe la continuité ? Que reste-t-il en particulier comme trace de l'échange, de la tension vers l'autre et des sentiments que son absence peut susciter? Si Balzac avait pu téléphoner à Madame Hanska ses Lettres à une étrangère auraient-elles seulement été écrites ? Pourrions-nous, plus de quatre-vingts ans après, partager l'émotion d'Apollinaire pensant à sa chère Lou s'il avait pu, «en temps réel », lui faire part de son désir et de ses rêveries ? Ce temps de la tension créatrice se donne à vivre dans l'arrêt, l'attente, le différé et la mise à distance. II est non superposable et non susceptible d'être dédoublé : l'individu y contient tout entier ${ }^{12}$.

Cette logique critique rappelle que, derrière l'apparence trompeuse d'un temps unique, universel et synchrone (celui des heures de la montre, des jours de l'agenda et des mois du calendrier), le temps est hétérogène. Qu'il n'y a pas un temps, mais des temps. Que la réalité du temps n'est pas seulement le temps réel. Que, face à l'entrée massive de notre société dans une culture de l'immédiat, de l'impulsion et de l'urgence généralisée, il y a des moments qui résistent à l'accélération, des durées qui ne sauraient être brusquées, et des instants qui échappent à la logique du gain et de la vitesse. Ces moments, ces durées et ces instants sont indispensables à la formation de soi comme sujet,

\footnotetext{
${ }^{12}$ Pour une généalogie de cette temporalité individuelle, voir Helga Nowotny (1992) ; pour la « mise en intrigue de soi " grâce à ce dégagement temporel, voir Paul Ricoeur (1983).
} 
Francis JAUREGUIBERRY

c'est-à-dire comme acteur capable de construire sa vie de façon autonome. Cette prise de distance en regard de l'instant permet à l'individu de se retrouver et en particulier de vivre une certaine autonomie par rapport à son rôle de gestionnaire efficace que tout le monde (y compris une part de lui-même) s'accorde à lui voir jouer. Elle lui permet de se « regarder de l'extérieur » et de concevoir sa vie non seulement comme déterminée par son héritage et par son insertion dans un système d'interdépendance, mais aussi comme le produit de sa réflexivité et de sa liberté individuelle. L'objectivation du monde qu'opère la modernité concerne aussi l'individu en lui donnant une définition extérieure de ce qu'il est (statut, rôles qu'il doit jouer, ressources sociales auxquelles il a recours pour agir). Or (toute l'œuvre de Georg Simmel nous le montre de manière particulièrement saisissante), l'individu moderne ne parvient jamais à totalement coïncider avec cette objectivité-là. I/ est cela : sa capacité réflexive l'en informe sans contestation possible. Mais il se sent aussi autre chose : une partie de son être se dérobe, sans cesse rétive à être cernée par une quelconque objectivité. Ce «reste de soi profond» est vécu par l'individu comme intrinsèquement personnel, intérieur, irrécupérable. Cette scission entre objectivité et subjectivité le soumet à une tension dont il ne peut se défaire.

Cette tension, qui est au fondement même des modalités existentielles de la modernité, se trouve multipliée chez l'individu contemporain. La mise à distance du monde, qui a accompagné son objectivation et sa rationalisation, a permis de le rendre plus maîtrisable et contrôlable. Comme le souligne Giddens (1994), le développement technologique et organisationnel a délivré l'individu des dangers et craintes situationnelles d'autrefois. Mais l'approfondissement du caractère objectif de la vie, en désamorçant les réponses sociales "enchantées " qu'apportaient mythes et traditions au questionnement existentiel, est aussi celui de l'insécurité ontologique. Celle-ci conduit l'individu à un nécessaire travail de construction de son existence de telle sorte qu'elle puisse avoir un sens à ses propres yeux. En somme, en l'absence d'un traitement social, l'individu doit trouver en lui-même le sens de son existence et surtout en expérimenter l'efficience sous la forme d'un contentement et d'un sentiment de vérité. La radicalisation instrumentale du réel se traduit chez l'individu par un besoin de préserver sa propre subjectivité sous une forme d'authenticité de soi à soi et de soi aux autres. C'est précisément à cette préservation que renvoie la logique critique sous la forme d'un écart, d'un " quant-à-soi empêchant l'individu d'être totalement son rôle ou sa position, d'être son personnage social » (Dubet, 1994, p. 129). 
Les téléphones portables, outils du dédoublement et de la densification du temps : un diagnostic confirmé

Rapportée à la consommation médiatique, cette même logique critique est indispensable pour "faire sens" dans l'inflation d'informations qui nous submerge. Comme le remarque Edgar Morin (1981, p. 26), "l'excès d'information étouffe l'information quand nous sommes soumis au déferlement ininterrompu d'événements sur lesquels on ne peut méditer parce qu'ils sont aussitôt chassés par d'autres événements (...) Alors que l'information apporte forme aux choses, la surinformation nous plonge dans l'informe. » Pour pouvoir « donner forme » aux informations, une prise de distance est nécessaire. Pour cesser d'être étourdi par leur défilement, troublé par leurs couleurs changeantes et ébloui par l'éclat de leur nouveauté, il faut savoir prendre du recul, être capable d'arrêter leur déferlement. À cette seule condition, ces mêmes informations peuvent être rapportées à un système de représentations et de significations qui permet, par la comparaison, la confrontation et la vérification, de formuler une interprétation. Mais l'individu-zappeur, obsédé par le mieux, a tendance à faire défiler de plus en plus vite ce qui lui est proposé afin d'être sûr de faire «le bon choix» ou d'avoir "la bonne information». Tout, dans la stratégie économique et les tactiques publicitaires des opérateurs de réseaux et des fournisseurs de service est fait pour encourager ce « désir de mieux ». L'offre en réseaux d'information, en services interactifs et en machines à communiquer ne cesse de s'amplifier. Bien sûr, personne ne s'en plaint. Mais il n'est pas certain que chacun possède une culture de la modération, de la distance et de la réflexion capable de résister à cette offre en information et en communication $^{13}$.

\section{6- L'expérience temporelle du branché}

L'expérience du branché dans la gestion du temps est le produit d'une sorte de dialogue tendu entre deux logiques d'action, sachant que l'une ne saurait entièrement recouvrir l'autre. D'un côté, une logique de gains et de vitesse qui est celle de la connexion, de la mise en synchronie et de l'urgence. De l'autre, une logique critique qui est celle de l'aménagement d'un temps à soi, de prise de distance et de déconnexion.

\footnotetext{
${ }^{13}$ Sur ce sujet, l'analyse de Gérard Claisse est bien pessimiste : “ À l'interconnexion en temps réel de nos prothèses informationnelles, répond la déconnexion généralisée de nos capacités à comprendre, à mettre en perspective, à contextualiser, à interpréter les informations qui nous parviennent. L'information entre, sort, circule, mais ne s'arrête pas. La prolifération d'informations nous rend aveugles. » (1997, pp. 123-124).
} 
Francis JAUREGUIBERRY

En 1996, je n'étais pas encore parvenu à théoriser cette tension comme un des éléments centraux de l'expérience du branché. J'en restais à la description de trois conduites : le refus, la fuite en avant, et la ruse (paragraphe 7). Ce n'est que quelques mois plus tard, après être parvenu à graphiquement représenter l'expérience du branché dans sa complexité (Jauréguiberry, 1997) que ces conduites m'apparurent dans toute leur signification ${ }^{14}$. Lorsque l'expérience du branché est trop fortement soumise à la logique de gains, d'immédiateté et de vitesse, elle s'effondre et menace de tomber dans le tourbillon du zappeur. La volonté, lorsqu'elle existe, d'y échapper donne généralement naissance à une conduite de fuite en avant. Voyant qu'il se fait posséder par une accélération non maîtrisée, le "branché dépassé " nourrit l'espoir qu'une gestion encore plus rentabiliste de son temps va lui permettre d'échapper au tourbillon. Son exaspération communicationnelle se traduit alors souvent par une exacerbation de son désir technique. À l'affût des dernières découvertes, il pense qu'une meilleure couverture du réseau, une plus grande intelligence de transmission, de nouveaux terminaux multifonctions, lui rendront un peu de temps et lui permettront de se retrouver. Soumettant (consciemment ou pas) leur vie à une logique de rentabilisation du temps, ceux qui adoptent cette attitude n'ont souvent pas d'autre choix que cette fuite en avant.

Lorsque l'expérience du branché est, à l'inverse, entièrement dominée par la logique de distanciation, elle conduit à une exigence d'authenticité et souvent à un enfermement subjectiviste dont le «temps à soi " constitue l'horizon. La déconnexion totale des technologies de l'information et de la communication (TIC) est une conduite extrême et très rare. Mais elle existe : sans pour cela se convertir en ermites, ceux qui «se retirent » se renferment sur leur foyer, leur maison, leur jardin... Ils cherchent (je reprends leurs termes) une "nouvelle simplicité », une « vérité de vie », une capacité à « être pleinement là ». Mais la majorité ne peut pas se déconnecter totalement. La pression économique et sociale est tout simplement trop forte. La tentation est alors grande de cliver les

\footnotetext{
14 II serait ici trop long de décrire cette expérience. Notons simplement qu'elle peut être représentée comme un cercle sur lequel agissent les forces attractives correspondant à trois logiques d'action (intégration, utilitaire et critique) auxquelles correspondent trois types d'action (être branché, être efficace, être autonome) et qui se donne à voir sous trois conduites archétypales : zapper, filtrer et préserver. Plus le branché se situe au centre du cercle, et plus il est en position de vivre une pleine expérience : sa position d'équidistance lui permet de ne pas être trop violemment attiré par l'une ou l'autre des forces. Au contraire, plus il est proche de la circonférence, et plus son action a de chances d'être aspirée par l'attraction d'une force ou par la résultante de deux. L'attraction de la force opposée (sur le cercle de l'expérience) peut dans certains cas être si faible que le branché se retrouve déséquilibré au point de tomber dans une situation qu'il ne maîtrise plus. II quitte alors expérience, happé par l'attraction de l'une ou l'autre des logiques d'action.
} 
Les téléphones portables, outils du dédoublement et de la densification du temps : un diagnostic confirmé

communications. D'un côté les communications « rentables », intéressées et stratégiques pour lesquelles la rapidité concourt à leur réussite, et de l'autre les communications «gratuites", intersubjectives et conviviales pour lesquelles le temps n'est pas compté. D'un côté, la communication « utile » et de l'autre la « vraie communication ». Les télécommunications nomades sont associées à la première, dans leur capacité même à organiser la seconde qui, elle, se fait (encore ?) en face-à-face.

La fuite en avant (connexion continue, hyper équipement, abandon de soi dans l'intensité) et le refus (déconnexion totale, burn out) sont des conduites de rupture avec l'expérience du branché. Les deux relèvent finalement d'une impossibilité ou d'une incapacité de conjuguer le désir ou la nécessité d'être «branché » avec la volonté de préserver un temps à soi. Une pleine expérience de «branché » renvoie au contraire à la nature hétérogène des logiques d'action qui la déterminent : utilitaire d'un côté, guidée par un souci de performance, d'efficacité et de gain, et critique de l'autre, motivée par la volonté de ne pas soumettre l'ensemble de sa vie à une mise en synchronie constante.

II s'ensuit des conduites de compromis, de ruse, de fines tactiques permettant de conjuguer au mieux ces deux exigences. Elles consistent pour l'essentiel à instaurer des filtres intelligents entre le réseau (la mise en synchronie) et l'acteur (recul réflexif, temps à soi). II s'agit de garder le contact sans en devenir l'esclave. Ces filtres sont généralement une tierce personne (en particulier le secrétariat sur le lieu de travail et le conjoint ou les enfants au domicile), un système mécanique de mise en différé (boîte vocale du portable, beeper, répondeur enregistreur et fax) ou un système de filtre en temps réel (répondeur enregistreur). Le succès des SMS et du courrier électronique s'inscrit dans cette logique. Une autre forme de mise à distance, que l'on pourrait appeler "repos du guerrier », consiste à se déconnecter de façon régulière mais éphémère afin de se « requinquer». Cette attitude est assez perverse puisqu'elle permet d'encaisser stress et urgence sans pour cela remettre en cause le mode de fonctionnement du système qui les produit. Ce sont par exemple les demi-heures de zen ou de "respirations profondes" organisées par l'entreprise pour ses employés ou de petits stages de déconnexion. "On travaille tous trop, me disait un cadre d'une start up de la Silicon Valley, on est tous workaholics, alors, de temps en temps, on fait des petits stages de désintoxication ». Et l'un des principes essentiels de ces stages consiste précisément à être totalement déconnecté. Tous les ans, le festival Burning man a lieu, durant une semaine, dans le désert du Nevada. En 2005, ils 
Francis JAUREGUIBERRY

étaient plus de 30000 à passer une semaine sans portable, "déconnectés mais branchés sur tous ceux qu'ils croisaient ". II est intéressant de noter que ce sont des techniciens, des ingénieurs et des managers qui fréquentent majoritairement ces lieux, avec quelques utopistes, évidemment. Finalement, lorsque la vie devient trop stressante, l'urgence trop pressante, on zappe sur un petit stage de déconnexion pour se recharger...

Bien sûr, l'équilibre est précaire et doit sans cesse être rétabli. II est des situations ou des périodes où le tourbillon du branché attire plus que d'autres où la volonté critique de prise de distance et de préservation d'une temporalité individuelle l'emporte. Mais c'est précisément parce qu'il n'existe pas de solution définitive en la matière que l'on peut parler, à propos de ceux qui se livrent à ce travail de mise en cohérence, d'acteurs sociaux en train de définir un nouvel enjeu social : le droit à la déconnexion.

\section{7- Le droit à la déconnection}

En conclusion de l'article, j'écrivais que le dédoublement du temps n'était vécu positivement que s'il était volontaire, et que, dans tous les autres cas, il risquait d'être subi comme une contrainte, un contrôle, un harcèlement, une source de stress. J'en déduisais :

II y a donc de fortes chances pour que la société informationnelle qui se dessine sous nos yeux connaisse une nouvelle catégorie (s'ajoutant aux autres) de riches et de pauvres. Les premiers occuperont une position les autorisant à pouvoir se déconnecter, les seconds, non. L'inaccessibilité devient inégalitaire. Pour l'instant, seule une minorité de la population vit cette expérience d'ubiquité médiatique. Et cette minorité est précisément composée de ceux (cadres supérieurs et dirigeants) qui possèdent le plus de ressources organisationnelles et de moyens culturels pour ne pas se laisser happer par le tourbillon du zappeur. Mais il n'est pas interdit de penser que, le «branchement universel " s'étendant à une proportion grandissante de la population (en particulier à des catégories professionnelles mises en situation de "harcèlement télécommunicationnel » par leur hiérarchie), le « droit à la déconnexion » devienne un enjeu social (p. 184). 
Les téléphones portables, outils du dédoublement et de la densification du temps : un diagnostic confirmé

La distinction que j'opérais il y a dix ans entre ceux ont le pouvoir de se déconnecter et ceux ont le devoir de rester branchés est désormais partout observable. Elle se donne particulièrement à voir sous la forme de l'extension des notions de garde et d'astreinte à des catégories professionnelles restées jusqu'alors en dehors de ce phénomène de gestion omniprésente de l'urgence. Jusqu'à la généralisation des portables, seuls certains professionnels connaissaient en effet les " astreintes », terme par lequel on désigne le fait d'être constamment disponible, prêt à intervenir immédiatement en dehors des horaires habituels de travail : la nuit ou l'espace d'un week-end par exemple. Les inspecteurs de police, les pompiers, les médecins hospitaliers, bref les professionnels de l'urgence "classique" connaissaient bien ces temps d'astreinte durant lesquels il ne fallait pas qu'ils s'éloignent de leur lieu de travail ou qu'ils quittent leur Alphapage. Mais la banalisation des portables, couplée à l'extension d'une gestion à flux tendus et en temps réel dans des branches de plus en plus nombreuses de l'activité économique, est synonyme d'extension très peu contrôlée pour l'instant - de la nécessité de rester branché, à l'écoute de son entreprise en dehors de son temps de travail. Ainsi, sous prétexte de sécurité ou de concurrence, des catégories entières de cadres ont gracieusement été dotées par leur direction de portables qui permettent de les joindre en tout lieu et à toute heure. Cadres supérieurs, responsables de directions, lignes ou secteurs, ils sont le nerf de la guerre économique qui oppose leur entreprise à ses concurrentes. Ils n'en sont toutefois pas les dirigeants ou les responsables ultimes. Ils gèrent plutôt les décisions de la direction en fonction des aléas internes de l'entreprise et des exigences externes du marché. Sans cesse sur la brèche, go-betweens incontournables, l'agilité des télécommunications les met dans une situation d'écoute et de disponibilité constantes. En aval, les personnels dont ils sont responsables ont en effet de plus en plus tendance à se référer directement à eux avant de prendre une décision face à une situation imprévue. C'est la réaction : "en cas de problèmes, on téléphone à la hiérarchie » (effet parapluie). Or, ces problèmes, dans notre économie concurrentielle d'adaptabilité, tendent précisément à se multiplier... En amont, les dirigeants ont, de leur côté, de plus en plus tendance à se détacher des interpellations à très court terme au profit d'une distance réflexive. Véritables "cadres fusibles", ils supportent toute l'intensité des flux informationnels de l'entreprise. Cette mise sous tension permanente, qui empiète très largement sur la vie privée de ces cadres hyper communicants, explique sans doute pourquoi certains d'entre eux « fondent les plombs » (précisément) ou « craquent » brusquement sans raison apparente... 
Francis JAUREGUIBERRY

Un nombre grandissant d'artisans et de professionnels indépendants supportent une tension similaire. N'ayant pas les moyens économiques ou organisationnels de se payer un secrétariat qui pourrait les décharger de ce nouvel afflux informationnel incontournable, ils doivent gérer de plus en plus de choses en simultané ou dans l'urgence. Le phénomène touche encore plus fortement les personnels nomades qui sont désormais contrôlés à distance par leur hiérarchie. Jusqu'alors en effet, l'autonomie des employés nomades devait beaucoup à la disjonction entre leur temps et leurs espaces de travail. Parce que physiquement éloignés de tout contrôle, ils pouvaient se livrer à des "aménagements personnels " de leur temps de travail, pourvu que les tâches attendues soient effectuées. Ils prenaient connaissance, en début de journée, du travail à effectuer puis commençaient leur tournée ou bien partaient sur leurs lieux d'intervention où ils demeuraient maîtres de leur temps. À partir du moment où il est possible de les surveiller en temps réel, la distance n'est plus synonyme de liberté. Au contraire: parce qu'éloignés, il semble bien qu'ils soient désormais davantage contrôlés! L'exemple type en la matière est celui des routiers : grâce au GPS et au portable, leur siège (hiérarchie gestionnaire) est constamment informé de l'avancement de leur travail. Le routier continue certes de gérer son temps, mais en se sachant observé, ce qui lui enlève une grande part de liberté. Jusqu'alors, il devait, en tant que salarié, parvenir à la meilleure synthèse possible entre, d'une part son désir de réaliser son travail le plus agréablement possible (ou, en tout cas, le moins péniblement: par exemple en s'aménageant des pauses, en prenant le temps de discuter avec les personnes rencontrées ou en décidant de son trajet) et, d'autre part, la nécessité de réaliser la tâche pour laquelle il était payé dans le temps imparti par le patron. II pouvait ainsi fort bien décider de s'arrêter deux heures au lieu d'une heure et de conduire ensuite six heures sans interruption... II était maître à bord. La rationalité de sa direction (tendant à toujours plus d'efficacité par une accélération des rotations) était «interprétée » par le routier, traduite à sa mesure. C'est cette gestion propre qui semble disparaître. II ne s'agit plus pour lui de faire un plan de route et de l'adapter selon sa forme, son humeur ou les aléas de la route, mais de suivre des directives précises, sans cesse réactualisées et auxquelles il doit se soumettre. En particulier, il n'est pas rare qu'un routier se voie désormais détourné pour prendre en compte un chargement " plus urgent». Le "siège » mesure sans doute mal le degré de désappointement que de tels changements, trop souvent répétés, peuvent produire. De planificateurs de temps impartis et de tâches à accomplir, les chauffeurs deviennent des fonctions programmables à distance. Leur capacité 
Les téléphones portables, outils du dédoublement et de la densification du temps : un diagnostic confirmé

d'improvisation et la fierté même d'exercer le métier de routier s'en trouvent atteintes. À trop vouloir fonctionnaliser et rentabiliser ainsi leurs employés en déplacement, les directions courent le risque de les voir se désinvestir d'une part de leurs responsabilités. Derrière la perte ou, tout au moins, la diminution d'autonomie de l'employé nomade, c'est le rapport de force entre deux logiques d'action qui se trouve modifié. D'une part, celle de l'employé, qui vise à avoir le plus de liberté possible pour gérer son emploi du temps et, d'autre part, celle de la direction qui vise à mieux contrôler ses employés distants afin de rendre leur travail plus efficace et leur déplacements plus rentables. D'un côté donc, une logique d'autonomie, de maîtrise et d'initiative dans le travail, de l'autre une logique de branchement, de réactivité immédiate et de prescriptions.

Pour l'heure, cette nouvelle forme de contrainte est surtout individuellement vécue. Le « cadre fusible », l'artisan ou l'employé nomade est d'abord confronté à lui-même. Dans certains cas même, il y a comme une intériorisation de la contrainte organisationnelle présentée... comme une liberté individuelle (celle, par exemple, de pouvoir rester chez soi durant les gardes... tant que tout va bien). Le coût social du stress, de l'énervement ou du malaise provoqués par cette nouvelle donne dans l'entreprise n'est collectivement pas mesurable. II semble renvoyer à des problèmes d'ordre purement individuel ou même psychologique. Pourtant une main invisible agit bien et les liens de dépendance renvoient bien à des formes de pouvoir, de domination et donc à des problèmes collectifs. Ce sont des catégories entières de personnel qui les connaissent désormais. II y a donc de fortes chances pour, qu'au cours des prochaines années, une prise de conscience se fasse autour de ce thème et qu'un nouveau droit en vienne à être revendiqué : le droit à la déconnexion et à l'isolement, le droit de refuser de porter un beeper jour et nuit ou bien d'être téléphoniquement partout et constamment joignable. Le droit à la dignité des personnes qui ne sauraient être réduites à être des fonctions ou des ressources contrôlables et corvéables à distance. Un droit dont l'application ne serait synonyme ni de sanction, ni de fuite, ni d'enfermement.

\section{Références bibliographiques}

AUBERT N., 2003, Le culte de l'urgence. La société malade du temps, Paris, Flammarion.

BAUMAN Z., 2003, La vie en miette. Expérience postmoderne et moralité, Rodez, Le Rouergue/Chambon. 
BAUMAN Z., 2004, L'amour liquide : de la fragilité des liens entre les hommes, Rodez, Le Rouergue/Chambon.

CLAISSE G., 1997, L'abbaye des télémythes, Lyon, Aléas.

DUBET F., 1994, Sociologie de l'expérience, Paris, Le Seuil.

EHRENBERG A., 1995, L'individu incertain, Paris, Calmann-Lévy.

GIDDENS A., 1994, Les conséquences de la modernité, Paris, l'Harmattan.

HEIDEGGER M., 1986, Être et temps, Paris, Gallimard.

JAURÉGUIBERRY F., 1996, « De l'usage des téléphones portatifs comme expérience du dédoublement et de l'accélération du temps ", Technologies de l'information et Société (TIS), vol. 8, n², pp. 169-187.

JAURÉGUIBERRY F., 1997, «L'usage du téléphone portable comme expérience sociale », Réseaux, n82-83, pp. 149-164.

JAURÉGUIBERRY F., 2000, « Mobile telecommunications and the management of time », Social Science Information, vol. 39, n², pp. 255-268.

JAURÉGUIBERRY F., 2003, Les branchés du portables. Sociologie des usages, Paris, Presses Universitaires de France.

LAÏDI Z., 1994, Un monde privé de sens, Paris, Fayard.

LAIIDI Z., 2000, Le sacre du présent, Paris, Flammarion.

LIPOVETSKY G., 1983, L'ère du vide, Paris, Gallimard.

LIPOVETSKY G., 1987, L'empire de l'éphémère, Paris, Gallimard.

MONTAIGNE M., 1965, Essais, Paris, Presses Universitaires de France.

MORIN E., 1981, Pour sortir du XXe siècle, Paris, Nathan.

NOWOTNY H., 1992, Le temps à soi. Genèse et structuration d'un sentiment du temps, Paris, Maison des sciences de l'homme.

RICOEUR P., 1983, Temps et récit. Tome I: L'intrigue et le récit historique, Le Seuil.

Pour citer cet article :

Jauréguiberry, F., «Les téléphones portables, outils du dédoublement et de la densification du temps : un diagnostic confirmé», Revue tic\&société [En ligne], Numéros, Volume 1, n9, 2ème semestre 2007, mis à jour le : 15/02/2008, URL : http://revues.mshparisnord.org/lodel/ticsociete/index.php?id=281>. 